\title{
Rapid repurposing of drugs for COVID-19
}

\author{
By R. Kiplin Guy', Robert S. DiPaola ${ }^{2}$, Frank Romanelli', Rebecca E. Dutch ${ }^{2}$
}

${ }^{1}$ College of Pharmacy, University of Kentucky, Lexington, KY, USA. ${ }^{2}$ College of Medicine, University of Kentucky, Lexington, KY, USA. Email: kip.guy@uky.edu

\section{The emergence of a new coronaviral respiratory disease calls for repurposing existing drugs}

In late fall 2019, a novel acute respiratory disease, called coronavirus disease 2019 (COVID-19) emerged in Wuhan, China. COVID-19 is caused by severe acute respiratory syndrome-coronavirus 2 (SARS-CoV-2) (1,2). COVID-19 has been declared a pandemic by the World Health Organization and continues to spread across the globe. Most patients recover within 1 to 3 weeks. However, a small proportion ( $5 \%)$ develop severe illness that can progress to acute respiratory distress syndrome (ARDS), which can lead to death. Currently, only supportive care is available; patients would greatly benefit from the availability of direct therapeutic approaches. One approach to identifying therapeutics is to repurpose approved drugs developed for other uses, which takes advantage of existing detailed information on human pharmacology and toxicology to enable rapid clinical trials and regulatory review.

The coronaviruses are single-stranded RNA viruses that infect vertebrates and move between different host species (3). With the emergence of SARS-CoV-2, there are now seven coronaviruses that are known to infect humans. Four of them (HCov-229E, HCoV-OC43, HCoV-NL63, and HCoV-HKU1) are responsible for $\sim 30 \%$ of cases of the common cold in humans. Two of them caused recent epidemics that had considerable associated mortality: SARS-CoV-1, which emerged in 20022003 and causes $\sim 10 \%$ mortality, and Middle East respiratory syndrome coronavirus (MERS-CoV), which emerged in 2012, is still active, and causes $\sim 35 \%$ mortality. Both epidemics affected a relatively small number of patients compared with COVID-19, which is more transmissible for several reasons, including asymptomatic carriers, long latency period, and high infectivity. Before COVID-19, only SARS-CoV-1 and MERS-CoV caused severe disease. Therefore, coronaviral drug discovery has been a small effort relative to that for other viral diseases such as influenza. Given the rapid spread of COVID-19 and its relatively high mortality, filling the gap for coronavirus-specific drugs is urgent.

The coronavirus life cycle (see the figure) involves a number of potentially targetable steps, including endocytic entry into host cells [involving angiotensin-converting enzyme 2 (ACE2) and transmembrane protease serine 2 (TMPRSS2)], RNA replication and transcription [involving helicase and RNA-dependent RNA polymerase (RdRp)], translation and proteolytic processing of viral proteins (involving chymotrypsin-like and papain-like proteases), virion assembly, and release of new viruses through the exocytic systems (4). In addition to virally encoded targets, numerous host targets are essential for viral replication and disease progression (3).

The cellular receptor for SARS-CoV-2 is ACE2 (5). Recombinant human ACE2 (rhACE2, or APNO1) is currently under development as a treatment for acute lung injury and pulmonary arterial hypertension and has proven well tolerated in a phase 1 trial in healthy volunteers. rhACE2 has been shown to significantly reduce viral entry into human cell-derived organoids (6), presumably by acting as a decoy for virus binding. This has lent support to the clinical trials that are investigating blockade of viral entry with APNO1 for COVID19 patients. Successful viral entry requires proteolytic processing of the viral coat spike glycoprotein (S), which can be carried out by TMPRSS2 (7). The TMPRSS2 inhibitor camostat (7) is approved in Japan for the treatment of chronic pancreatitis and postoperative gastric reflux and is generally well tolerated, although rare serious side effects have been reported. Both camostat and the related agent nafamostat (8) block SARS-CoV-2 replication in TMPRSS2-expressing human cells. Camostat has been shown to block infection with SARS-CoV-2 in a mouse model. Therefore, there is a strong rationale to support clinical trials with these drugs for COVID-19, which have already been initiated in the Netherlands and Germany.

Coronaviruses use the endo-lysosomal pathway to enter the cell before uncoating. Chloroquine (CQ) and hydroxychloroquine (HCQ) are antimalarial drugs that affect endosomal function and block autophagosome-lysosome fusion (9). Both drugs have been shown to inhibit SARS-CoV-2 replication in cellular models $(8,10)$. Azithromycin (AZ), a widely used broad-spectrum antibiotic, also blocks autophagosome clearance in human cells (11) and replication of the Zika virus and influenza virus in human cells in vitro (12). Preliminary results from a small randomized trial of HCQ in COVID-19 patients report a reduction in time to clinical resolution (13). A small open-label trial has demonstrated increased reduction in viral load for COVID-19 patients receiving the combination of HCQ and AZ relative to HCQ alone, although this study has been heavily criticized because of post hoc removal of several subjects from the study analysis (14). These hypothesis- 
generating studies have justified emergency approval of their use for COVID-19 in the United States, where they are both being widely used.

However, both HCQ and AZ have potential cardiac toxicity (QT prolongation, which can lead to fatal arrhythmia), and HCQ additionally has the potential for negative effects on the eye. Understanding risk-benefit ratios is paramount if these drugs are to become a standard of care for COVID-19. Several post hoc analyses carried out in the United States and Europe suggest modest benefit, at best, from HCQ monotherapy for COVID-19 patients; one large post hoc analysis among U.S. veterans suggests that there is harm to patients from HCQ. Given the mechanistic rationale but lack of well-designed clinical studies and potential for drug-induced toxicity, there is a key need for controlled, randomized trials to test the efficacy and safety of these drugs for COVID-19 patients.

After uncoating, the viral genomic RNA is used for capdependent translation to produce two polypeptides, which are then autoproteolytically processed to produce several viral proteins, including RdRp and two proteases. Although the proteases might seem attractive targets given the number of viral protease inhibitors previously developed for HIV and other viruses, they are only distantly related to other viral proteases. The combination of the HIV protease inhibitors lopinavir and ritonavir (15) proved clinically ineffective for COVID-19 patients, as had previously been the case for the same combination in SARS-CoV-1 disease. Therefore, further repurposing with this class of drugs is poorly justified-although there are other protease inhibitors in early-stage drug discovery that are directed to the coronavirus proteases.

Production of the replication complex proteins, including the helicase and RdRp, allows for genomic replication of the virus and for production of subgenomic RNAs, which are also translated to produce structural and coat proteins. The helicase is theoretically an attractive target, but it is divergent from other viral helicases, and there is no evidence that the herpes simplex virus helicase inhibitors amenamevir or pretelivir are effective against coronaviruses.

RdRp carries out both replication and transcription of the viral RNA, making it a clear target for blocking the viral life cycle. Because RdRp is a critical protein for many viruses, a number of broad-spectrum RdRp inhibitors are either approved or in clinical trials, including remdesivir and favipiravir. Remdesivir was initially developed to treat the flaviviruses that cause Ebola and Marburg diseases and has proven safe in trials during the past two Ebola epidemics. However, it is less effective for Ebola than antibody-based treatments that prevent the virus from entering human cells. Remdesivir was subsequently shown to be active against both SARS-CoV-1 and MERS-CoV in animal models. Favipiravir was developed for influenza and approved in Japan in 2014, specifically for new pandemic influenza outbreaks. Both remdesivir and favipiravir are active against SARS-CoV-2 in human cells in vitro (7). Remdesivir has been rapidly advanced into several clinical trials for COVID-19, and early informal data being released from those trials suggest that remdesivir is effective, but such data sets need to be used cautiously for generalizing the understanding of either safety or efficacy. Further randomized, controlled trials with RdRp inhibitors are justified and needed.

The best justified drugs for repurposing to treat COVID19 patients are the host-factor-targeted drugs HCQ, AZ, and camostat and nafamostat and the viral RdRp-targeted drugs remdesivir and favipiravir. A number of other drugs are also being considered, although with less supporting evidence (see supplementary materials). Additionally, phenotypic screening approaches are being developed on the basis of either viral entry or replication that could be used to survey approved drugs and drug candidates much more widely. Both of these approaches may widen the available classes of drugs for consideration.

The key issue with any of these potential treatments is to balance the oppositional needs of making treatment decisions for individual patients during epidemic peaks on the basis of clinical studies that involve small numbers of patients with ensuring that well-designed, randomized clinical trials are carried out rapidly to provide proof that they are safe and efficacious. COVID-19 is expected to be active permanently and several seasons of disease peaks are likely before herd (population) immunity is established. The difficulty is to coordinate rapid hypothesis-generating studies during this first peak to justify a smaller number of well-controlled large trials to be executed in later peaks to provide the data needed for approval of drugs for COVID-19. Researchers, ethics boards, and regulators are accustomed to developing trial plans over months, not weeks-a time frame that is not afforded during this emergent situation. It is necessary for all involved to work faster and more efficiently and then position the well-justified drugs for registration-enabling trials during the next peak.

\section{REFERENCES AND NOTES}

1. C. del Rio, P. N. Malani, JAMA 323, 1339 (2020). doi:10.1001/jama.2020.3072

2. N. Zhu et al.; China Novel Coronavirus Investigating and Research Team, N. Engl. J. Med. 382, 727 (2020). doi:10.1056/NEJMoa2001017Medline

3. T. S. Fung, D. X. Liu, Annu. Rev. Microbiol. 73, 529 (2019). doi:10.1146/annurevmicro-020518-115759 Medline

4. G. Li, E. De Clercq, Nat. Rev. Drug Discov. 19, 149 (2020). doi:10.1038/d41573-02000016-0 Medline

5. J. Lanet al., Nature 10.1038/s41586-020-2180-5 (2020).

6. V. Monteil et al., Cell 10.1016/j.cell.2020.04.004 (2020).

7. M. Hoffmannet al., Cell 10.1016/j.cell.2020.02.052 (2020).

8. M. Wang et al., Cell Res. 30, 269 (2020). doi:10.1038/s41422-020-0282-0 Medline

9. M. Mautheet al., Autophagy 14, 1435 (2018). doi:10.1080/15548627.2018.1474314 Medline

10. J. Liu et al., Cell Discov. 6, 16 (2020). doi:10.1038/s41421-020-0156-0 Medline 
11. M. Rennaet al., J. Clin. Invest. 121, 3554 (2011). doi:10.1172/JCI46095 Medline

12. D. H. Tran et al., J. Antibiot. (Tokyo) 72, 759 (2019). doi:10.1038/s41429-0190204-x Medline

13. Z. Chen et al., medRxiv, 2020.03.22.20040758 [Preprint]. 10 April 2020. https://www.medrxiv.org/content/10.1101/2020.03.22.20040758v3.

14. P. Gautret et al., Int. J. Antimicrob. Agents 105949 (2020). 10.1016/i.jiantimicag.2020.105949 Medline

15. B. Cao et al., N. Engl. J. Med. 10.1056/NEJMoa2001282 (2020).

\section{ACKNOWLEDGMENTS}

The authors acknowledge the participation of the COVID-19 Unified Research Experts (CURE) Alliance team at the University of Kentucky in discussions underlying this piece. We have no funding supporting the writing of this article and no conflicts of interest. The therapeutics discussed in this Perspective are undergoing clinical testing and are not currently approved for the treatment of COVID-19.

\section{SUPPLEMENTARY MATERIALS}

science.sciencemag.org/cgi/content/full/science.abb9332/DC1

Published online 8 May 2020

10.1126/science.abb9332 


\section{Possible targets in the coronavirus life cycle}

This simplified coronavirus life cycle shows the processes and proteins that could be therapeutically targeted with existing drugs that have the potential to be repurposed for the treatment of COVID-19.

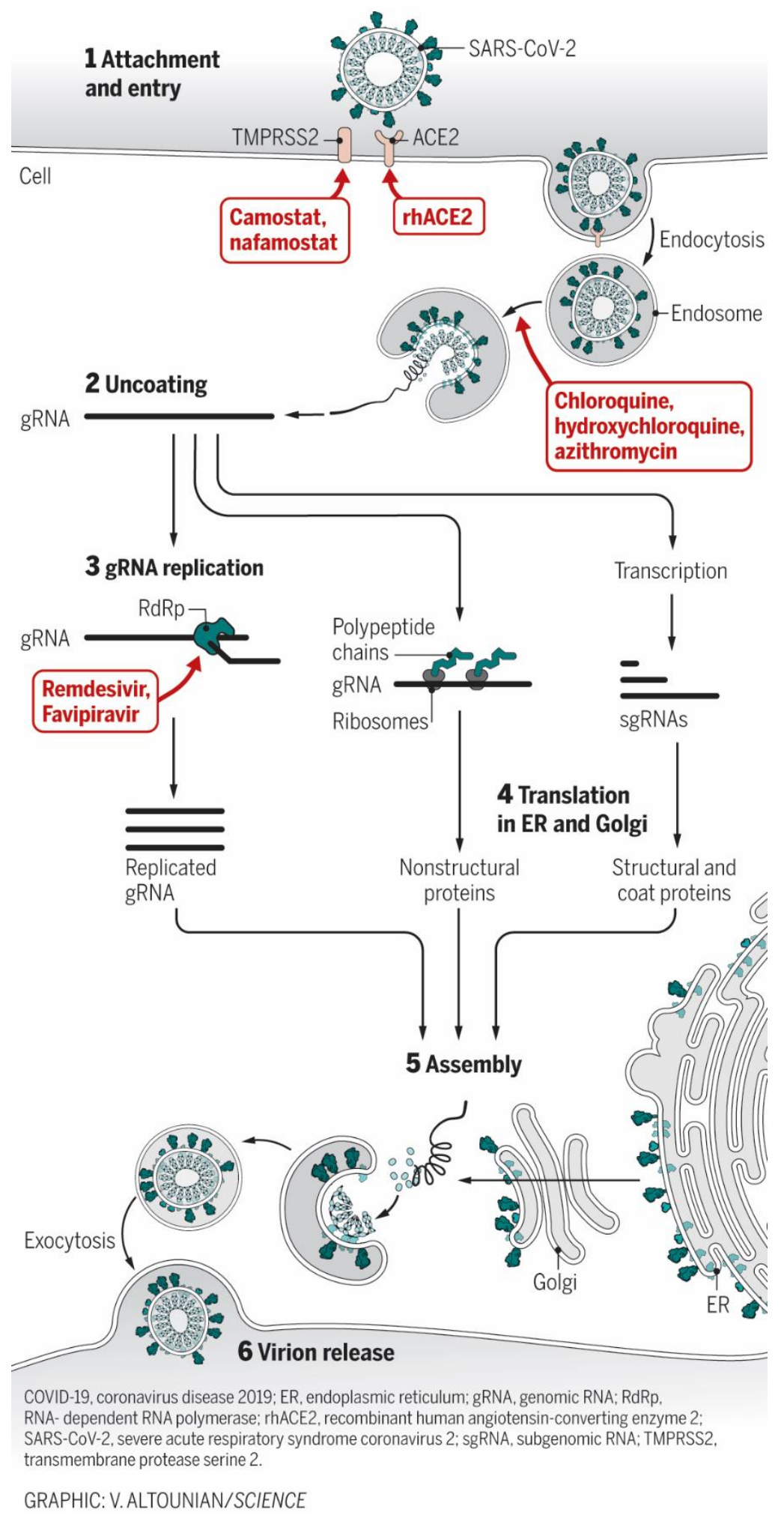


